\title{
A Versatile and General One-pot Method for Synthesising bis- Spiroketal Motifs
}

Thomas Georgiou, Maria Tofi, Tamsyn Montagnon and Georgios Vassilikogiannakis*

Department of Chemistry, University of Crete, 71409 Iraklion, Crete, Greece

vasil@chemistry.uoc.grT

\section{Contents:}

(1) Experimental procedures for synthesis of all relevant compounds: pages 2 - 8

(2) Copies of Relevant Spectra: pages 9 - 20 


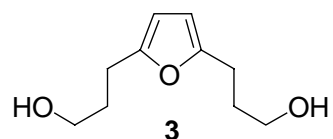

To a solution of ester $2(231 \mathrm{mg}, 0.96 \mathrm{mmol})$ in anhydrous $\mathrm{Et}_{2} \mathrm{O}(4 \mathrm{~mL})$ under an argon atmosphere at $0{ }^{\circ} \mathrm{C}$, was added $\mathrm{LiAlH}_{4}(73 \mathrm{mg}, 1.92 \mathrm{mmol})$. The reaction was then warmed to room temperature and stirred for $30 \mathrm{~min}$ after which it was partitioned between $\mathrm{Et}_{2} \mathrm{O}(10 \mathrm{~mL})$ and $\mathrm{NH}_{4} \mathrm{Cl}(10 \mathrm{~mL})$. The layers were separated and the organic phase dried $\left(\mathrm{Na}_{2} \mathrm{SO}_{4}\right)$ and concentrated in vacuo to afford the desired dihydroxyfuran 3 (145 mg, $82 \%)$.

${ }^{1} \mathrm{H}$ NMR $\left(500 \mathrm{MHz}, \mathrm{CDCl}_{3}\right): \delta=5.86(\mathrm{~s}, 2 \mathrm{H}), 3.64(\mathrm{t}, J=6.4 \mathrm{~Hz}, 4 \mathrm{H}), 2.65(\mathrm{t}, J=7.4$ $\mathrm{Hz}, 4 \mathrm{H}), 2.23$ (brs, -OH), 1.85 (m, 4H) ppm; ${ }^{13} \mathrm{C}$ NMR (125 MHz, $\left.\mathrm{CDCl}_{3}\right): \delta=153.9$ (2C), 105.4 (2C), $62.0(2 \mathrm{C}), 31.0$ (2C), 24.4 (2C) ppm.

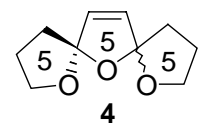

Diol 3 (70 mg, $0.38 \mathrm{mmol})$ was dissolved in $\mathrm{CH}_{2} \mathrm{Cl}_{2}(20 \mathrm{~mL})$ containing catalytic amounts $\left(10^{-4} \mathrm{M}\right)$ of Methylene Blue. The solution was cooled to $0{ }^{\circ} \mathrm{C}$. Oxygen was bubbled gently through the solution while it was irradiated with light from a xenon Variac Eimac Cermax $300 \mathrm{~W}$ lamp for $2 \mathrm{~min}$. Dimethyl sulfide (278 $\mu \mathrm{L}, 3.80 \mathrm{mmol})$ was added and the solution warmed to room temperature after which it was stirred for a further $12 \mathrm{~h}$. The solvent was removed in vacuo and the residue passed through a short pad of silica (hexane:EtOAc $=4: 1)$ to afford $4(55 \mathrm{mg}, 80 \%)$ as a 1:1 mixture of stereoisomers.

${ }^{1} \mathrm{H}$ NMR (500 MHz, $\mathrm{CDCl}_{3}$, mixture of two stereoisomers): $\delta=5.93$ (s, 2H), 5.92 (s, 2H), $4.14(\mathrm{~m}, 2 \mathrm{H}), 4.08(\mathrm{~m}, 2 \mathrm{H}), 3.87(\mathrm{~m}, 4 \mathrm{H}), 2.15(\mathrm{~m}, 6 \mathrm{H}), 1.97(\mathrm{~m}, 10 \mathrm{H}) \mathrm{ppm} ;{ }^{13} \mathrm{C}$ NMR (125 MHz, $\mathrm{CDCl}_{3}$ ): $\delta=132.9$ (2C), 132.6 (2C), 117.1 (2C), 116.2 (2C), 68.7 (2C), $68.4(2 \mathrm{C}), 36.8$ (2C), 36.4 (2C), 24.9 (2C), 24.8 (2C) ppm. 


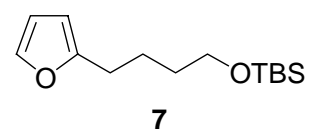

To a solution of $\mathrm{n}-\mathrm{BuLi}$ (3.14 $\mathrm{mL}$ of $1.4 \mathrm{M}$ solution in Hexane, $4.4 \mathrm{mmol}$ ) in anhydrous THF (2 mL) at $-25{ }^{\circ} \mathrm{C}$, a solution of furan $5(300 \mathrm{mg}, 4.4 \mathrm{mmol})$ in anhydrous THF (2 $\mathrm{mL})$ was added dropwise. After $4 \mathrm{~h}$ stirring at $-15{ }^{\circ} \mathrm{C}$, a solution of iodide $\mathbf{6}^{16}(690 \mathrm{mg}$, $2.2 \mathrm{mmol})$ in anhydrous THF $(3 \mathrm{~mL})$ was added dropwise and the reaction mixture stirred for $1 \mathrm{~h}$ at $-15{ }^{\circ} \mathrm{C}$. The reaction was next warmed to room temperature and stirred for a further $4 \mathrm{~h}$ after which it was then partitioned between $\mathrm{Et}_{2} \mathrm{O}(15 \mathrm{~mL})$ and $\mathrm{H}_{2} \mathrm{O}(15 \mathrm{~mL})$. The layers were separated and the organic layer was washed with brine $(15 \mathrm{~mL})$, dried $\left(\mathrm{Na}_{2} \mathrm{SO}_{4}\right)$, and concentrated in vacuo. The residue was purified by column chromatography ( furan 7 (505 mg, $90 \%)$.

${ }^{1} \mathrm{H}$ NMR $\left(500 \mathrm{MHz}, \mathrm{CDCl}_{3}\right): \delta=7.30(\mathrm{~d}, J=1.7 \mathrm{~Hz}, 1 \mathrm{H}), 6.28\left(\mathrm{dd}, J_{1}=2.9 \mathrm{~Hz}, J_{2}=1.7\right.$ $\mathrm{Hz}, 1 \mathrm{H}), 5.98(\mathrm{~d}, J=2.9 \mathrm{~Hz}, 1 \mathrm{H}), 3.63(\mathrm{t}, J=6.5 \mathrm{~Hz}, 2 \mathrm{H}), 2.65(\mathrm{t}, J=7.4 \mathrm{~Hz}, 2 \mathrm{H}), 1.69$ $(\mathrm{m}, 2 \mathrm{H}), 1.57$ (m, 2H), 0.90 (s, 9H), 0.05 (s, 6H) ppm; ${ }^{13} \mathrm{C} \mathrm{NMR}\left(125 \mathrm{MHz}, \mathrm{CDCl}_{3}\right): \delta=$ 156.3, 140.7, 110.0, 104.7, 62.8, 32.3, 27.7, 25.9 (3C), 24.4, 18.3, -5.3 (2C) ppm; HRMS (TOF ES+): calcd for $\mathrm{C}_{14} \mathrm{H}_{26} \mathrm{O}_{2} \mathrm{NaSi}$ : $277.1600[\mathrm{M}+\mathrm{Na}]^{+}$; found: 277.1591.

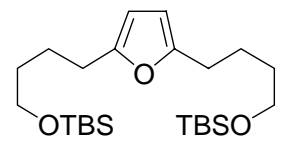

To a solution of monosubstituted furan $7(200 \mathrm{mg}, 0.79 \mathrm{mmol})$ in anhydrous THF (4 mL) at $0{ }^{\circ} \mathrm{C}, n$-BuLi $(0.84 \mathrm{~mL}$ of $1.4 \mathrm{M}$ solution in hexane, $1.18 \mathrm{mmol})$ was added dropwise. After $20 \mathrm{~min}$ stirring at the same temperature, a solution of iodide 6 (371 mg, $1.18 \mathrm{mmol})$ in anhydrous THF ( $4 \mathrm{~mL})$ was added dropwise. The reaction was then warmed to room temperature and stirred for a further $3.5 \mathrm{~h}$ after which it was partitioned between $\mathrm{Et}_{2} \mathrm{O}(10$ $\mathrm{mL})$ and $\mathrm{NH}_{4} \mathrm{Cl}(10 \mathrm{~mL})$. The layers were separated and the organic layer washed brine 
$(10 \mathrm{~mL})$, dried $\left(\mathrm{Na}_{2} \mathrm{SO}_{4}\right)$, and concentrated in vacuo to afford the desired symmetric bisTBS protected dihydroxyfuran $(300 \mathrm{mg}, 86 \%)$.

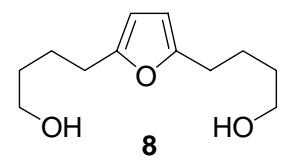

To a solution of bis-TBS protected dihydroxyfuran $(300 \mathrm{mg}, 0.68 \mathrm{mmol})$ in anhydrous THF $(6 \mathrm{~mL})$ at $0{ }^{\circ} \mathrm{C}$, TBAF $(1.71 \mathrm{~mL}$ of $1.0 \mathrm{M}$ solution in THF, $1.71 \mathrm{mmol})$ was added dropwise. The reaction mixture was then warmed to room temperature and stirred for $2 \mathrm{~h}$ after which it was partitioned between $\mathrm{Et}_{2} \mathrm{O}(15 \mathrm{~mL})$ and $\mathrm{H}_{2} \mathrm{O}(15 \mathrm{~mL})$. The layers were separated and the organic phase was dried $\left(\mathrm{Na}_{2} \mathrm{SO}_{4}\right)$ and concentrated in vacuo. The residue was purified by flash column chromatography ( 1:1) to afford dihydroxy furan 8 (114 mg, $80 \%)$.

${ }^{1} \mathrm{H}$ NMR $\left(500 \mathrm{MHz}, \mathrm{CDCl}_{3}\right): \delta=5.86(\mathrm{~s}, 2 \mathrm{H}), 3.65(\mathrm{t}, J=6.5 \mathrm{~Hz}, 4 \mathrm{H}), 2.61(\mathrm{t}, J=7.3$ $\mathrm{Hz}, 4 \mathrm{H}), 1.69(\mathrm{~m}, 4 \mathrm{H}), 1.61(\mathrm{~m}, 4 \mathrm{H}) \mathrm{ppm} ;{ }^{13} \mathrm{C} \mathrm{NMR}\left(125 \mathrm{MHz}, \mathrm{CDCl}_{3}\right): \delta=154.1(2 \mathrm{C})$, 105.3 (2C), 62.6 (2C), 32.1 (2C), 27.7 (2C), 24.4 (2C) ppm.

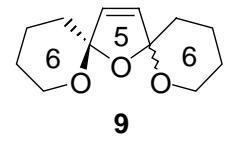

Dihydroxyfuran $8(50 \mathrm{mg}, 0.23 \mathrm{mmol})$ was dissolved in $\mathrm{CH}_{2} \mathrm{Cl}_{2}(15 \mathrm{~mL})$ containing catalytic amounts $\left(10^{-4} \mathrm{M}\right)$ of Methylene Blue. The solution was cooled to $0{ }^{\circ} \mathrm{C}$. Oxygen was gently bubbled through the solution while it was irradiated with a xenon Variac Eimac Cermax $300 \mathrm{~W}$ lamp for $2 \mathrm{~min}$. Dimethyl sulfide $(168 \mu \mathrm{L}, 2.30 \mathrm{mmol})$ was added and the solution was warmed to room temperature and stirred for a further $12 \mathrm{~h}$. The solvent was removed in vacuo and the residue passed through a short pad of silica (hexane:EtOAc $=2: 1)$ to afford bis-spioketal $9(37 \mathrm{mg}, 77 \%)$ as a 55:45 mixture of diastereoisomers (Please note that before column chromatography bis-spiroketal 9 existed as a 3:1 mixture of stereoisomers). 
${ }^{1} \mathrm{H}$ NMR (500 MHz, $\mathrm{C}_{6} \mathrm{D}_{6}$, mixture of two stereoisomers): $\delta=5.73$ (s, 2H), $5.72(\mathrm{~s}, 2 \mathrm{H})$, $4.07(\mathrm{~m}, 4 \mathrm{H}), 3.70(\mathrm{~m}, 4 \mathrm{H}), 1.95(\mathrm{~m}, 4 \mathrm{H}), 1.83(\mathrm{~m}, 2 \mathrm{H}), 1.64\left(\mathrm{dd}, J_{1}=13.0 \mathrm{~Hz}, J_{2}=4.3\right.$ $\mathrm{Hz}, 2 \mathrm{H}), 1.60(\mathrm{~m}, 2 \mathrm{H}), 1.47(\mathrm{~m}, 8 \mathrm{H}), 1.24(\mathrm{~m}, 6 \mathrm{H}) \mathrm{ppm} ;{ }^{13} \mathrm{C} \mathrm{NMR}\left(125 \mathrm{MHz}, \mathrm{C}_{6} \mathrm{D}_{6}\right.$, mixture of two stereoisomers): $\delta=134.7(2 \mathrm{C}), 134.6(2 \mathrm{C}), 110.4(2 \mathrm{C}), 109.4(2 \mathrm{C}), 63.7$ (2C), $63.6(2 \mathrm{C}), 36.2(2 \mathrm{C}), 35.8(2 \mathrm{C}), 26.1$ (4C), 20.6 (4C) ppm.

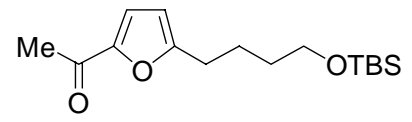

To a solution of monosubstituted furan $7(1.0 \mathrm{~g}, 3.93 \mathrm{mmol})$ in anhydrous THF $(10 \mathrm{~mL})$, at $0{ }^{\circ} \mathrm{C}, n$-BuLi ( $4.2 \mathrm{~mL}$ of $1.4 \mathrm{M}$ solution in hexane, $5.88 \mathrm{mmol}$ ) was added dropwise. After $20 \mathrm{~min}$ stirring at the same temperature, a solution of $N$-methoxy- $N$ methylacetamide $(605 \mathrm{mg}, 5.88 \mathrm{mmol})$ in anhydrous THF $(7 \mathrm{~mL})$ was added dropwise. The reaction mixture was warmed to room temperature and stirred for a further $3 \mathrm{~h}$ after which it was partitioned between $\mathrm{Et}_{2} \mathrm{O}(20 \mathrm{~mL})$ and $\mathrm{NH}_{4} \mathrm{Cl}(20 \mathrm{~mL})$. The layers were separated and the organic layer was washed with brine $(20 \mathrm{~mL})$, dried $\left(\mathrm{Na}_{2} \mathrm{SO}_{4}\right)$, and concentrated in vacuo. The residue was purified by flash column chromatography (silica gel, hexane:EtOAc $=20: 1 \rightarrow 10: 1)$ to afford the desired methyl furylketone $(0.97 \mathrm{~g}, 84$ $\%)$.

${ }^{1} \mathrm{H}$ NMR $\left(500 \mathrm{MHz}, \mathrm{CDCl}_{3}\right): \delta=7.07(\mathrm{~d}, J=3.5 \mathrm{~Hz}, 1 \mathrm{H}), 6.16(\mathrm{~d}, J=3.5 \mathrm{~Hz}, 1 \mathrm{H}), 3.62$ (t, $J=6.2 \mathrm{~Hz}, 2 \mathrm{H}), 2.71(\mathrm{t}, J=7.6 \mathrm{~Hz}, 2 \mathrm{H}), 2.42(\mathrm{~s}, 3 \mathrm{H}), 1.74(\mathrm{~m}, 2 \mathrm{H}), 1.56(\mathrm{~m}, 2 \mathrm{H})$, 0.89 (s, 9H), 0.03 (s, 6H) ppm; ${ }^{13} \mathrm{C}$ NMR (125 MHz, $\left.\mathrm{CDCl}_{3}\right): \delta=186.0,161.8,151.4$, 119.0, 108.1, 62.6, 32.1, 28.0, 25.9 (3C), 25.6, 24.1, 18.3, -5.4 (2C) ppm; HRMS (TOF ES+): calcd for $\mathrm{C}_{16} \mathrm{H}_{29} \mathrm{O}_{3} \mathrm{Si}: 297.1886[\mathrm{M}+\mathrm{H}]^{+}$; found: 297.1896 .

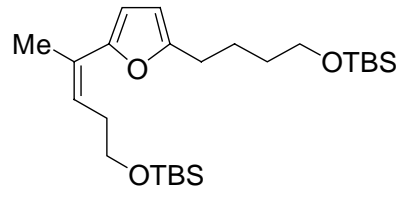


To a solution of phosphonium bromide $10(1.0 \mathrm{~g}, 1.94 \mathrm{mmol})$ in anhydrous THF $(7 \mathrm{~mL})$ at $-78{ }^{\circ} \mathrm{C}$, LiHMDS (1.94 $\mathrm{mL}$ of $1.0 \mathrm{M}$ solution in THF, $1.94 \mathrm{mmol}$ ) was added dropwise. After $1 \mathrm{~h}$ at the same temperature, a solution of previously synthesized methyl furylketone $(0.5 \mathrm{~g}, 1.70 \mathrm{mmol})$ in anhydrous THF $(3 \mathrm{~mL})$ was added dropwise and the reaction mixture stirred for a further $1 \mathrm{~h}$. The reaction was then warmed to room temperature over $2 \mathrm{~h}$ and stirred for a further $2.5 \mathrm{~h}$. Hexane $(40 \mathrm{~mL})$ was added to precipitate the byproduct $\left(\mathrm{PPh}_{3} \mathrm{O}\right)$ which was then removed by filtration. The solvent was evaporated in vacuo and the residue was purified by flash column chromatography (silica gel, hexane:EtOAc $=$ $20: 1)$ to afford the desired olefin $(0.58 \mathrm{~g}, 77 \%)$ as a mixture of geometric isomers ( $Z: E=$ 85:15, Z-configuration of major isomer confirmed by nOe).

${ }^{1} \mathrm{H}$ NMR (500 MHz, $\mathrm{C}_{6} \mathrm{D}_{6}, Z$-isomer): $\delta=6.14(\mathrm{~d}, J=3.2 \mathrm{~Hz}, 1 \mathrm{H}), 5.90(\mathrm{~d}, J=3.2 \mathrm{~Hz}$, 1H), 5.41 (brt, $J=6.9 \mathrm{~Hz}, 1 \mathrm{H}), 3.70(\mathrm{t}, J=6.8 \mathrm{~Hz}, 2 \mathrm{H}), 3.48(\mathrm{t}, J=6.3 \mathrm{~Hz}, 2 \mathrm{H}), 2.82$ (brq, $J=6.7 \mathrm{~Hz}, 2 \mathrm{H}), 2.50$ (t, $J=7.5 \mathrm{~Hz}, 2 \mathrm{H}), 1.97(\mathrm{~s}, 3 \mathrm{H}), 1.68(\mathrm{~m}, 2 \mathrm{H}), 1.48(\mathrm{~m}, 2 \mathrm{H})$, 0.97 (s, 9H), 0.95 (s, 9H), 0.06 (s, 6H), 0.03 (s, 6H) ppm; ${ }^{13} \mathrm{C}$ NMR $\left(125 \mathrm{MHz}, \mathrm{C}_{6} \mathrm{D}_{6}, \mathrm{Z}-\right.$ isomer): $\delta=156.1,154.4,126.4,124.5,109.9,107.2,64.0,63.5,34.2,33.2,28.8,26.8$ (3C), 26.7 (3C), 25.4, 22.5, 19.2, 19.1, -4.5 (2C), -4.6 (2C) ppm; HRMS (TOF ES+): calcd for $\mathrm{C}_{25} \mathrm{H}_{49} \mathrm{O}_{3} \mathrm{Si}_{2}: 453.3220[\mathrm{M}+\mathrm{H}]^{+}$; found: 453.3236 .

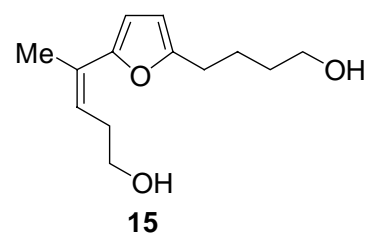

To a solution of olefin $(262 \mathrm{mg}, 0.58 \mathrm{mmol})$ in anhydrous THF $(6 \mathrm{~mL})$ at $0{ }^{\circ} \mathrm{C}$, TBAF $(1.45 \mathrm{~mL}$ of $1.0 \mathrm{M}$ solution in THF, $1.45 \mathrm{mmol})$ was added dropwise. The reaction mixture was then warmed to room temperature and stirred for a further $1.5 \mathrm{~h}$. The reaction mixture was then partitioned between $\mathrm{Et}_{2} \mathrm{O}(10 \mathrm{~mL})$ and $\mathrm{H}_{2} \mathrm{O}(10 \mathrm{~mL})$ and the layers were separated. The organic phase was dried $\left(\mathrm{Na}_{2} \mathrm{SO}_{4}\right)$ and concentrated in vacuo to afford dihydroxy furan $\mathbf{1 5}(108 \mathrm{mg}, 80 \%)$ as a mixture of geometric isomers ( $Z$ : $E=$ $85: 15)$.

${ }^{1} \mathrm{H}$ NMR (500 MHz, $\mathrm{C}_{6} \mathrm{D}_{6}, Z$-isomer): $\delta=6.11(\mathrm{~d}, J=3.1 \mathrm{~Hz}, 1 \mathrm{H}), 5.88(\mathrm{~d}, J=3.1 \mathrm{~Hz}$, 1H), 5.26 (brt, $J=7.4 \mathrm{~Hz}, 1 \mathrm{H}), 3.63(\mathrm{t}, J=7.2 \mathrm{~Hz}, 2 \mathrm{H}), 3.42$ (t, $J=6.4 \mathrm{~Hz}, 2 \mathrm{H}), 2.81$ 
(brq, $J=7.3 \mathrm{~Hz}, 2 \mathrm{H}), 2.46$ ( t $J=7.3 \mathrm{~Hz}, 2 \mathrm{H}), 1.91(\mathrm{~d}, J=0.7 \mathrm{~Hz}, 3 \mathrm{H}), 1.62(\mathrm{~m}, 2 \mathrm{H})$, 1.44 (m, 2H) ppm; ${ }^{13} \mathrm{C}$ NMR (125 MHz, $\mathrm{C}_{6} \mathrm{D}_{6}, \mathrm{Z}$-isomer): $\delta=156.3,154.5,126.6,124.0$, 109.8, 107.3, 63.1, 62.7, 34.3, 33.0, 28.7, 25.5, 22.5 ppm; HRMS (TOF ES+): calcd for $\mathrm{C}_{13} \mathrm{H}_{20} \mathrm{O}_{3} \mathrm{Na}: 247.1310[\mathrm{M}+\mathrm{Na}]^{+}$; found: 247.1302 .

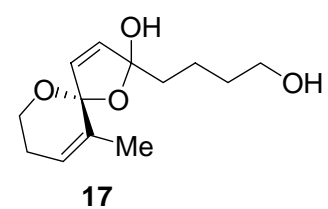

Diol $15(50 \mathrm{mg}, 0.22 \mathrm{mmol})$ was dissolved in $\mathrm{CH}_{2} \mathrm{Cl}_{2}(15 \mathrm{~mL})$ containing catalytic amounts $\left(10^{-4} \mathrm{M}\right)$ of Methylene Blue. The solution was cooled to $0{ }^{\circ} \mathrm{C}$. Oxygen was gently bubbled through the solution while it was irradiated with a xenon Variac Eimac Cermax $300 \mathrm{~W}$ lamp for $5 \mathrm{~min}$. Dimethyl sulfide $(161 \mu \mathrm{L}, 2.20 \mathrm{mmol})$ was added and the solution was warmed to room temperature and stirred for a further $1.5 \mathrm{~h}$. The solvent was removed in vacuo and the residue was chromatographed (silica gel, hexane:EtOAc $=1: 1$ $\rightarrow 1: 2$ ) to afford hemiketal $\mathbf{1 7}$ (40 $\mathrm{mg}, 88 \%$ based on only Z-isomer of diol 15 reacting) as a 2:1 mixture of spiroketal stereoisomers.

${ }^{1} \mathrm{H}$ NMR (500 MHz, $\mathrm{C}_{6} \mathrm{D}_{6}$, major stereoisomer, the ${ }^{1} \mathrm{H}$ NMR data for this compound is extremely sensitive to concentration): $\delta=5.99$ (d, $J=5.6 \mathrm{~Hz}, 1 \mathrm{H}$ ), 5.61 (d, $J=5.6 \mathrm{~Hz}$, $1 \mathrm{H}), 5.51(\mathrm{brd}, J=5.0 \mathrm{~Hz}, 1 \mathrm{H}), 4.01(\mathrm{~m}, 1 \mathrm{H}), 3.68\left(\mathrm{dd}, J_{1}=10.7 \mathrm{~Hz}, J_{2}=6.0 \mathrm{~Hz}, 1 \mathrm{H}\right)$, $3.53(\mathrm{t}, J=6.3 \mathrm{~Hz}, 2 \mathrm{H}), 2.04(\mathrm{~m}, 2 \mathrm{H}), 1.90(\mathrm{~m}, 2 \mathrm{H}), 1.70(\mathrm{~m}, 2 \mathrm{H}), 1.55$ (brs, 3H), 1.53 (m, 2H) ppm; ${ }^{13} \mathrm{C}$ NMR (125 MHz, $\mathrm{C}_{6} \mathrm{D}_{6}$, major stereoisomer): $\delta=137.3,133.8,132.7$, 125.3, 111.3, 108.6, 62.9, 60.9, 39.2, 33.8, 25.9, 21.3, 19.4 ppm; HRMS (TOF ES+): calcd for $\mathrm{C}_{13} \mathrm{H}_{20} \mathrm{O}_{4} \mathrm{Na}: 263.1259[\mathrm{M}+\mathrm{Na}]^{+}$; found: 263.1257.

${ }^{1} \mathrm{H}$ NMR (500 MHz, $\mathrm{C}_{6} \mathrm{D}_{6}$, minor stereoisomer, the ${ }^{1} \mathrm{H}$ NMR data for this compound is extremely sensitive to concentration): $\delta=5.93(\mathrm{~d}, J=5.6 \mathrm{~Hz}, 1 \mathrm{H}$ ), 5.65 (d, $J=5.6 \mathrm{~Hz}$, $1 \mathrm{H}), 5.55$ (brd, $J=4.9 \mathrm{~Hz}, 1 \mathrm{H}), 4.01(\mathrm{~m}, 1 \mathrm{H}), 3.75\left(\mathrm{dd}, J_{1}=10.7 \mathrm{~Hz}, J_{2}=5.9 \mathrm{~Hz}, 1 \mathrm{H}\right)$, 3.58 (t, $J=5.9 \mathrm{~Hz}, 2 \mathrm{H}), 2.04$ (m, 2H), 1.90 (m, 2H), 1.73 (brs, 3H), $1.70(\mathrm{~m}, 2 \mathrm{H}), 1.50$ (m, $2 \mathrm{H}) \mathrm{ppm} ;{ }^{13} \mathrm{C}$ NMR $\left(125 \mathrm{MHz}, \mathrm{C}_{6} \mathrm{D}_{6}\right.$, minor): $\delta=136.3,134.5,133.3,124.6,112.2$, 
109.3, 62.9, 60.8, 41.4, 33.6, 26.0, 21.5, 19.6 ppm; HRMS (TOF ES+): calcd for $\mathrm{C}_{13} \mathrm{H}_{20} \mathrm{O}_{4} \mathrm{Na}: 263.1259[\mathrm{M}+\mathrm{Na}]^{+}$; found: 263.1257 .

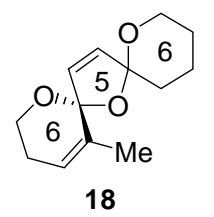

To a solution of previously prepared $17(39 \mathrm{mg}, 0.16 \mathrm{mmol})$ in anhydrous THF $(5 \mathrm{~mL})$, p-TsOH$\bullet \mathrm{H}_{2} \mathrm{O}(15 \mathrm{mg}, 0.08 \mathrm{mmol})$ was added and the solution stirred for $30 \mathrm{~min}$ at room temperature. A reaction solution was portioned between $\mathrm{Et}_{2} \mathrm{O}(5 \mathrm{~mL})$ and a saturated solution of $\mathrm{NaHCO}_{3}(5 \mathrm{~mL})$. The layers were separated and the organic phase washed with brine $(5 \mathrm{~mL})$ and dried $\left(\mathrm{Na}_{2} \mathrm{SO}_{4}\right)$. The solvent was removed in vacuo and the residue purified by column chromatography ( silica gel, hexane:EtOAc $=8: 1 \rightarrow 4: 1$ ) to afford bisspiroketal 18 (33 mg, $0.15 \mathrm{mmol}, 91 \%)$ initially as a 3:2 mixture of diastereoisomers, going to a 1:1 mixture upon repurification by column chromatography (silica gel).

${ }^{1} \mathrm{H}$ NMR $\left(500 \mathrm{MHz}, \mathrm{C}_{6} \mathrm{D}_{6}\right.$, diastereoisomer A): $\delta=5.80(\mathrm{~d}, J=5.7 \mathrm{~Hz}, 1 \mathrm{H}), 5.67(\mathrm{~d}, J=$ $5.7 \mathrm{~Hz}, 1 \mathrm{H}), 5.54$ (brs, 1H), 4.13 (dt, $\left.J_{1}=11.8 \mathrm{~Hz}, J_{2}=2.4 \mathrm{~Hz}, 1 \mathrm{H}\right), 4.05(\mathrm{~m}, 1 \mathrm{H}), 3.75$ (m, 2H), $2.09(\mathrm{~m}, 1 \mathrm{H}), 1.95(\mathrm{~m}, 1 \mathrm{H}), 1.63(\mathrm{~m}, 2 \mathrm{H}), 1.54$ (brs, 3H), 1.47 (m, 3H), 1.25 (m, 1H) ppm; ${ }^{13} \mathrm{C}$ NMR (125 MHz, $\mathrm{C}_{6} \mathrm{D}_{6}$, diastereoisomer $\left.\mathrm{A}\right): \delta=136.2,134.4,133.7,125.0$, 110.6, 109.6, 63.8, 60.8, 36.4, 34.6, 26.1, 20.5, 19.4 ppm; HRMS (TOF ES+): calcd for $\mathrm{C}_{13} \mathrm{H}_{18} \mathrm{O}_{3} \mathrm{Na}: 245.1154[\mathrm{M}+\mathrm{Na}]^{+}$; found: 245.1143 .

${ }^{1} \mathrm{H}$ NMR (500 MHz, $\mathrm{C}_{6} \mathrm{D}_{6}$, diastereoisomer B): $\delta=5.80(\mathrm{~d}, J=5.7 \mathrm{~Hz}, 1 \mathrm{H}), 5.64(\mathrm{~d}, J=$ $5.7 \mathrm{~Hz}, 1 \mathrm{H}), 5.54$ (brs, 1H), 4.05 (m, 2H), 3.75 (m, 1H), 3.67 (m, 1H), 2.09 (m, 1H), 1.95 $(\mathrm{m}, 1 \mathrm{H}), 1.81$ (brs, 3H), $1.62(\mathrm{~m}, 2 \mathrm{H}), 1.47(\mathrm{~m}, 3 \mathrm{H}), 1.25(\mathrm{~m}, 1 \mathrm{H}) \mathrm{ppm} ;{ }^{13} \mathrm{C}$ NMR $(125$ $\mathrm{MHz}, \mathrm{C}_{6} \mathrm{D}_{6}$, diastereoisomer B): $\delta=136.2,135.1,133.3,124.0,110.1,109.6,63.4,60.9$, 36.4, 34.6, 26.0, 20.6, 19.4 ppm; HRMS (TOF ES+): calcd for $\mathrm{C}_{13} \mathrm{H}_{18} \mathrm{O}_{3} \mathrm{Na}$ : 245.1154 [M $+\mathrm{Na}]^{+}$; found: 245.1143 . 
Copies of relevant ${ }^{1} \mathrm{H}$ NMR and ${ }^{13} \mathrm{C}$ NMR spectra:

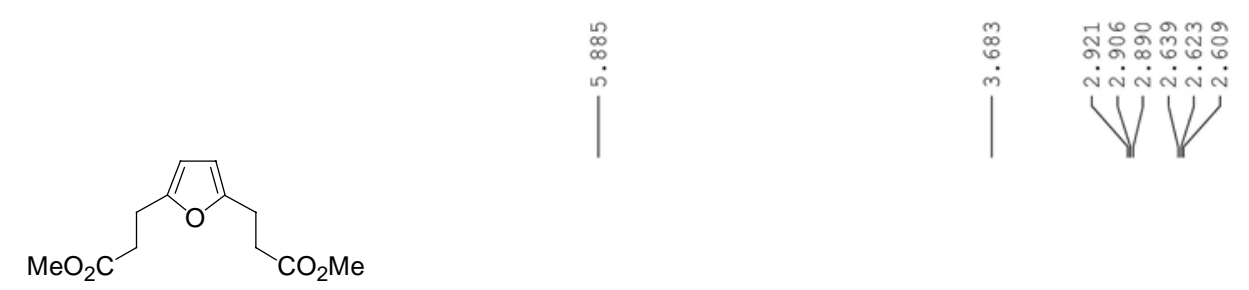

$\left(500 \mathrm{MHz}, \mathrm{CDCl}_{3}\right.$ )
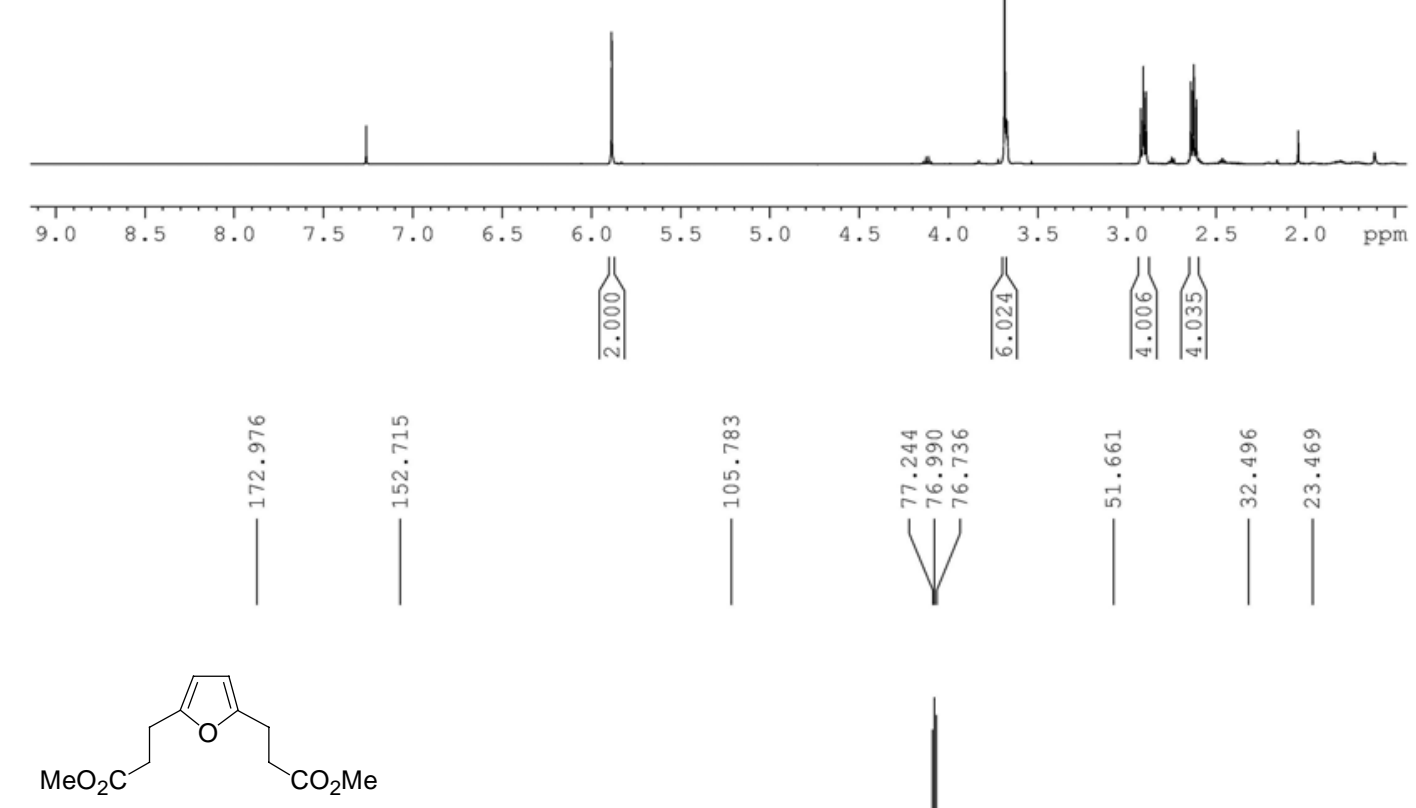

(125 MHz, $\mathrm{CDCl}_{3}$ )
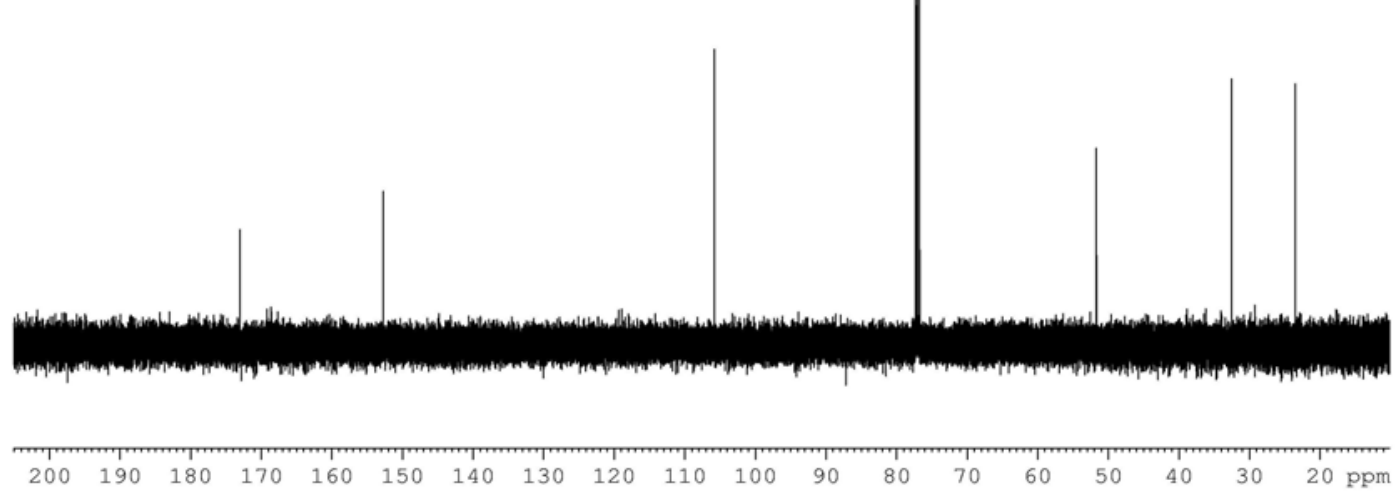


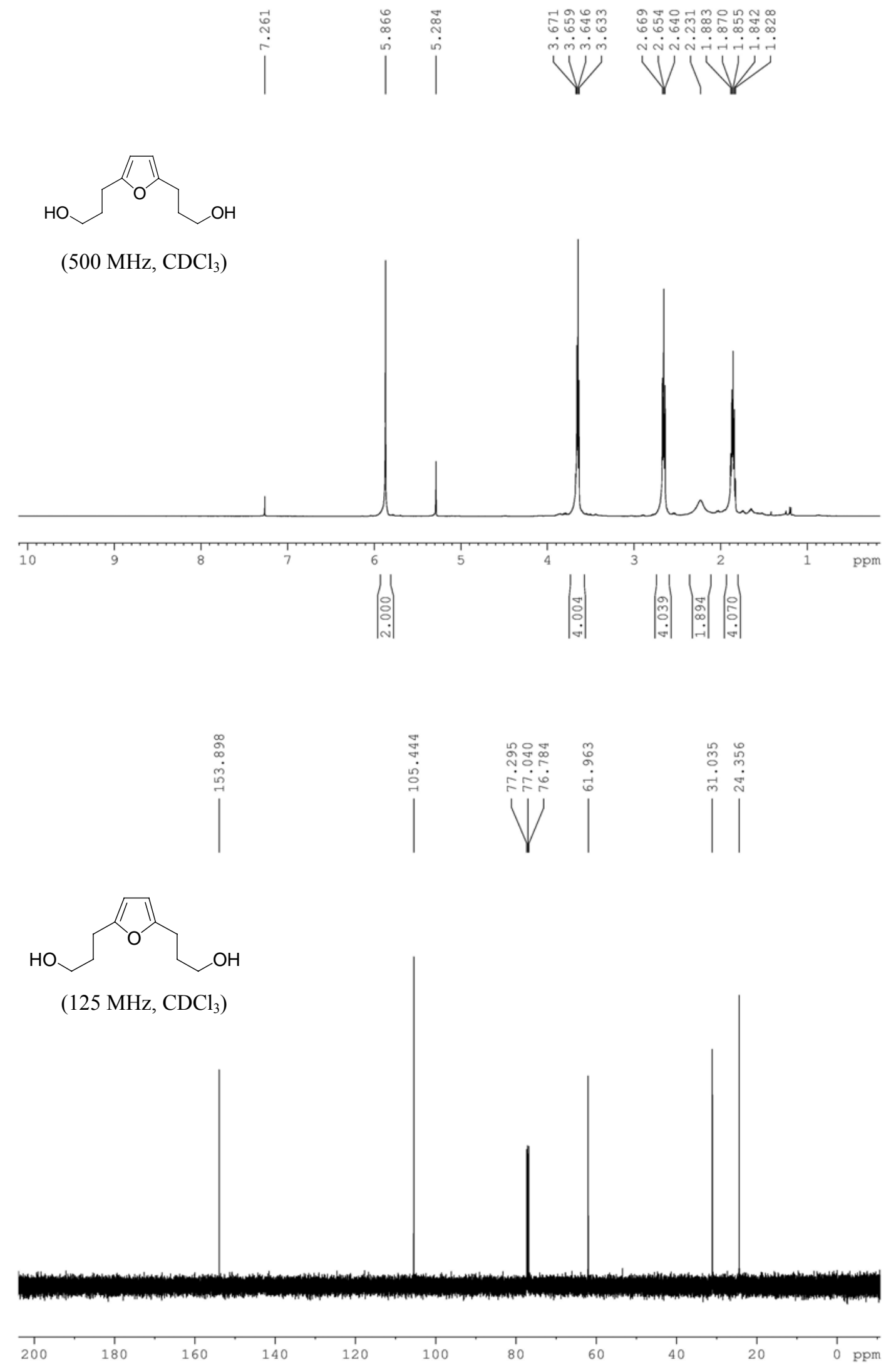




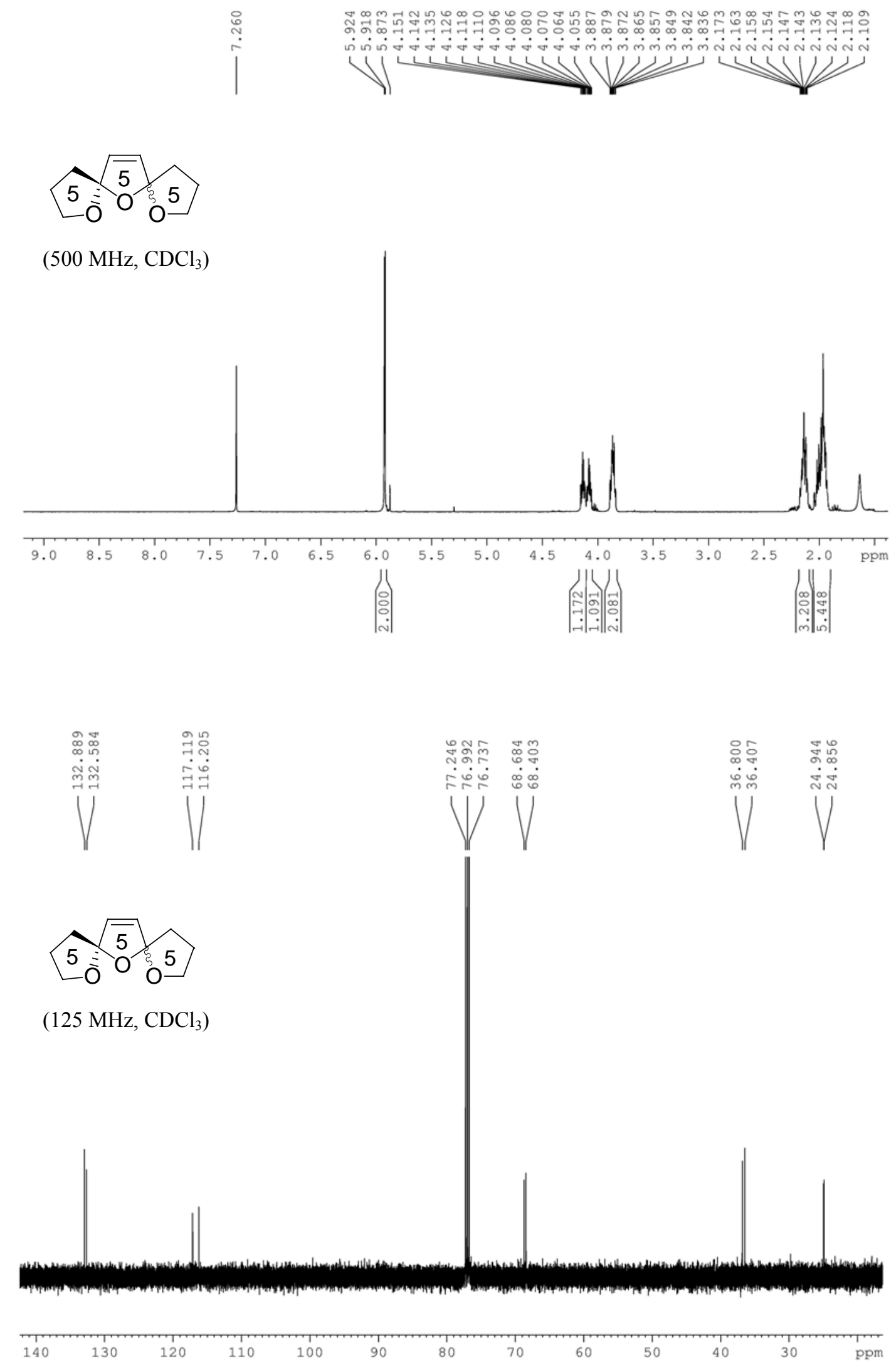




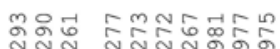

irit

$1 / 4$

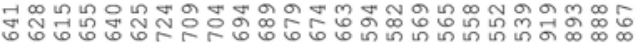

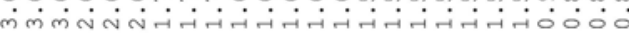

L1

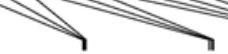

مOTBS

$\left(500 \mathrm{MHz}, \mathrm{CDCl}_{3}\right)$
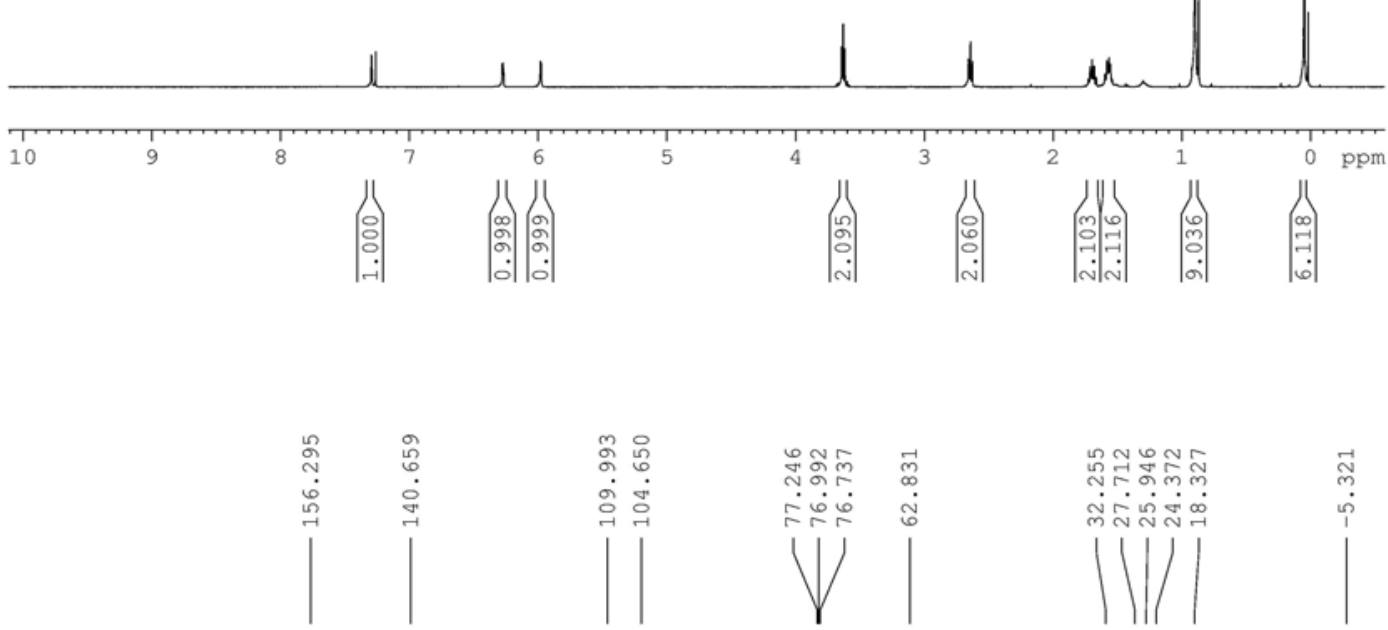

مOTBS

(125 MHz, $\mathrm{CDCl}_{3}$ )

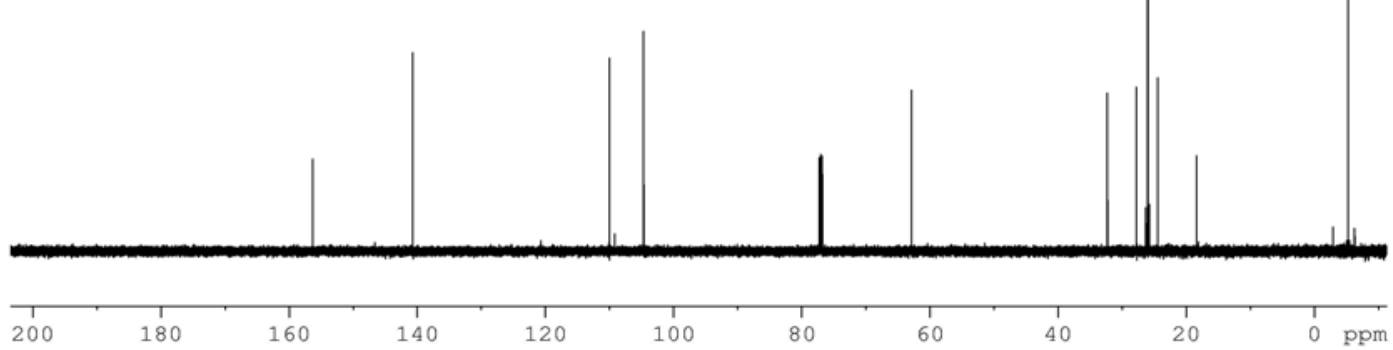



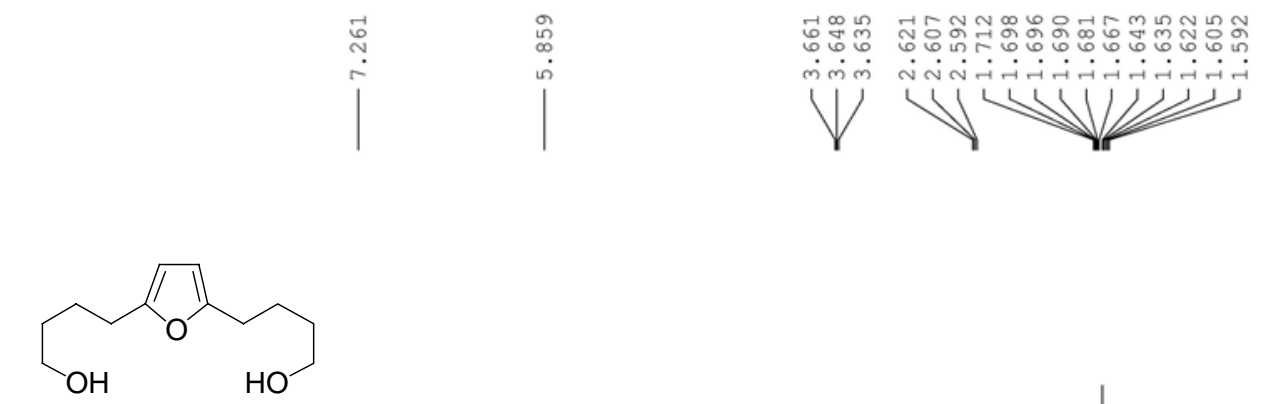

(500 MHz, $\mathrm{CDCl}_{3}$ )
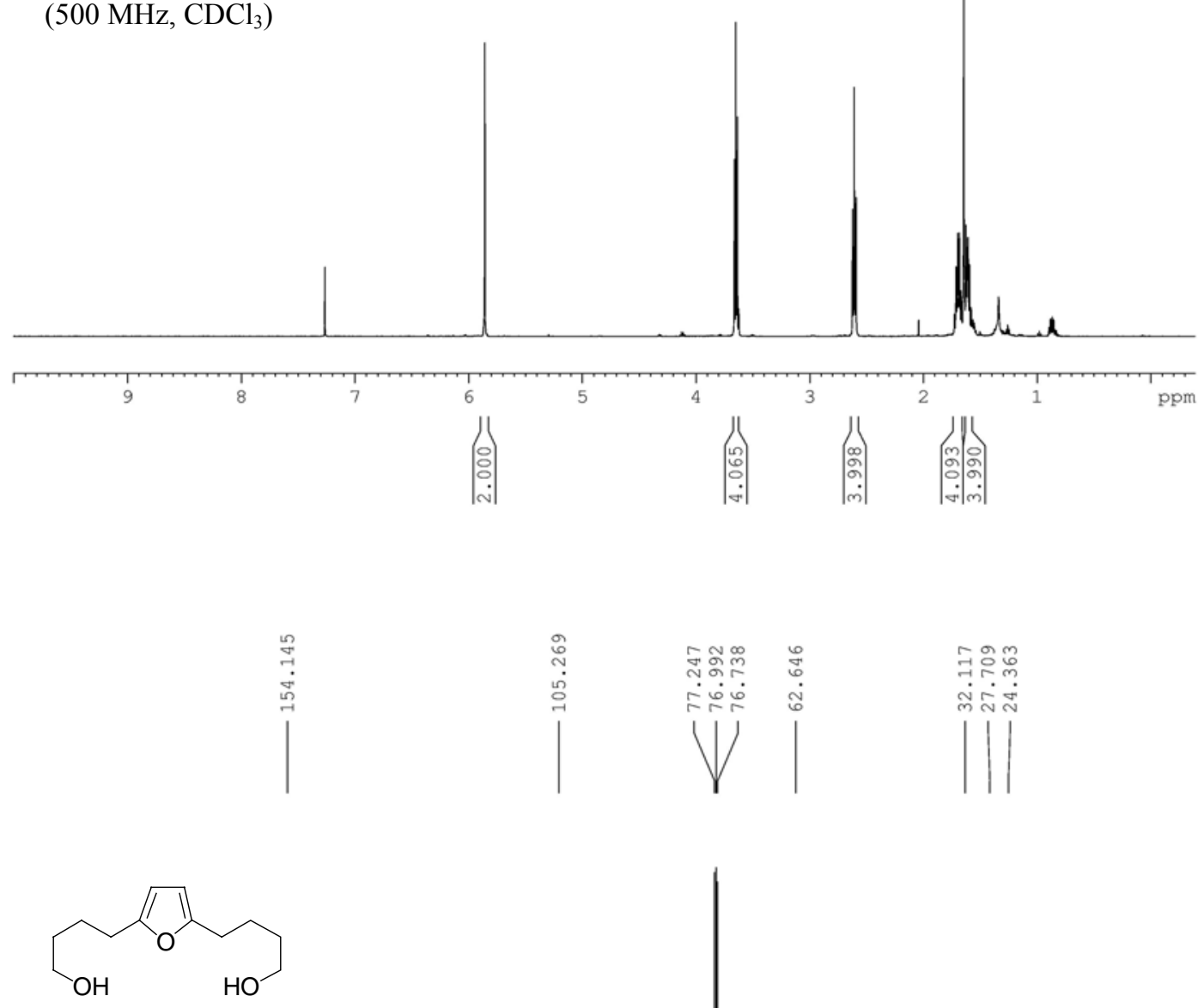

(125 MHz, $\mathrm{CDCl}_{3}$ )

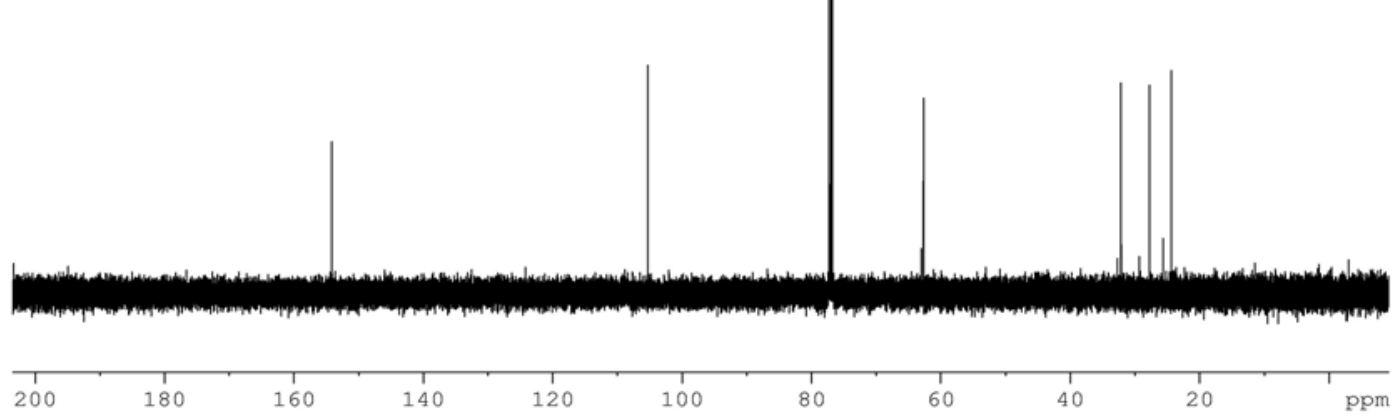




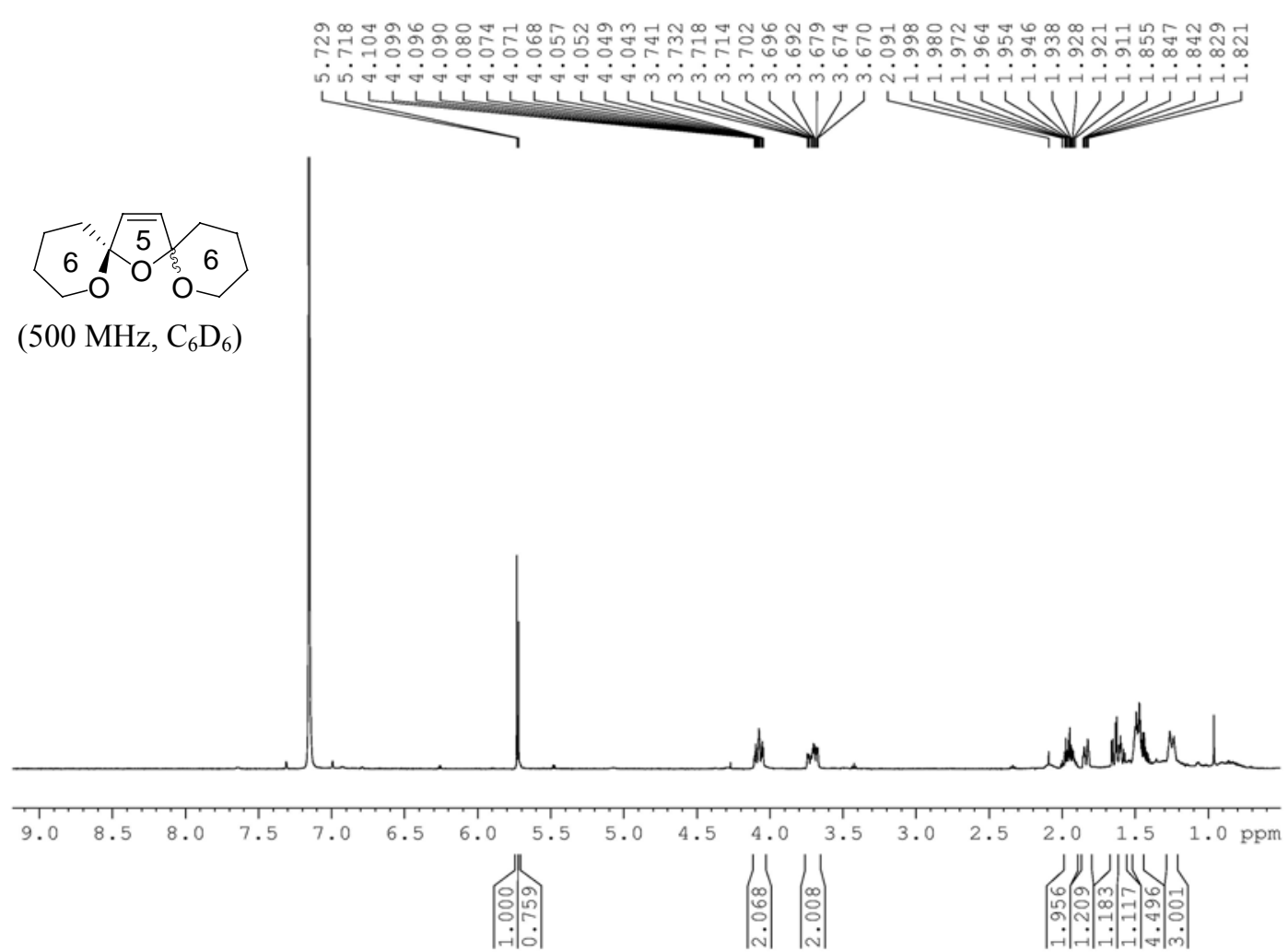

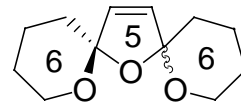
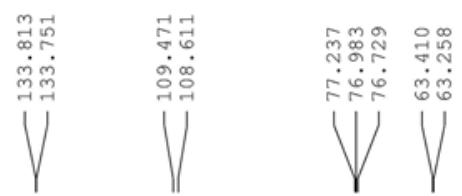

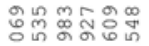

लिं लंखं

VV

(125 $\mathrm{MHz}, \mathrm{CDCl}_{3}$ )

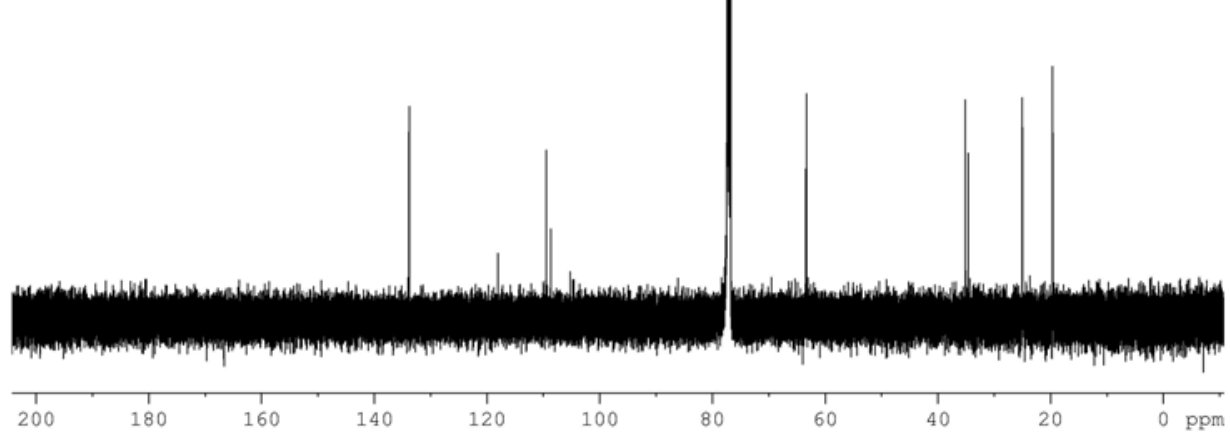




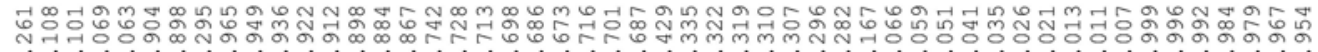

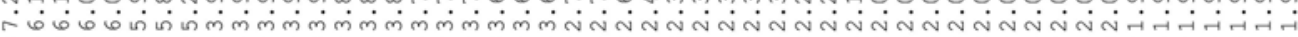
[था<smiles>CC1(c2ccc(CCCO)o2)CCCO1</smiles>

(500 $\mathrm{MHz}, \mathrm{CDCl}_{3}$ )
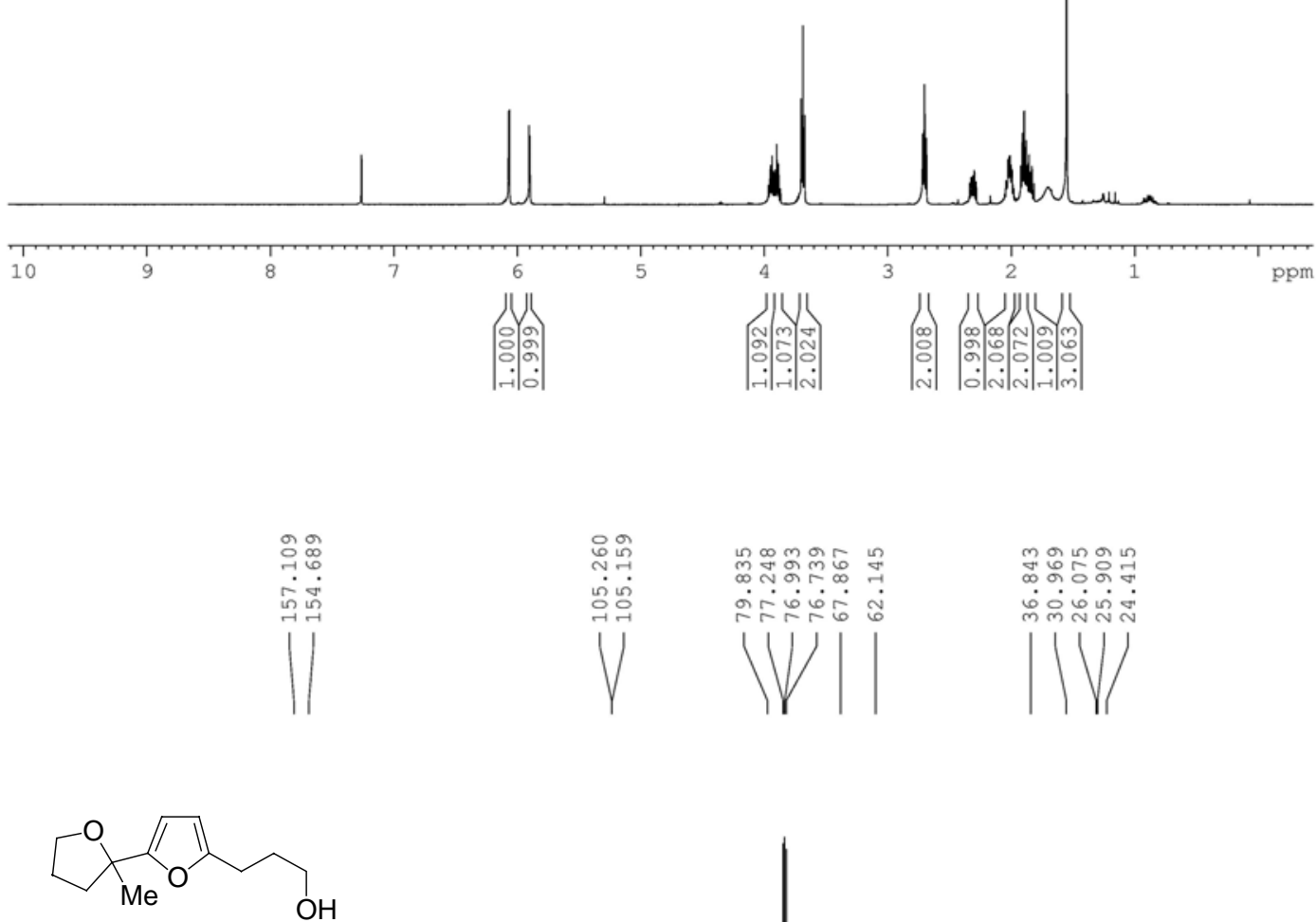

$\left(125 \mathrm{MHz}, \mathrm{CDCl}_{3}\right)$

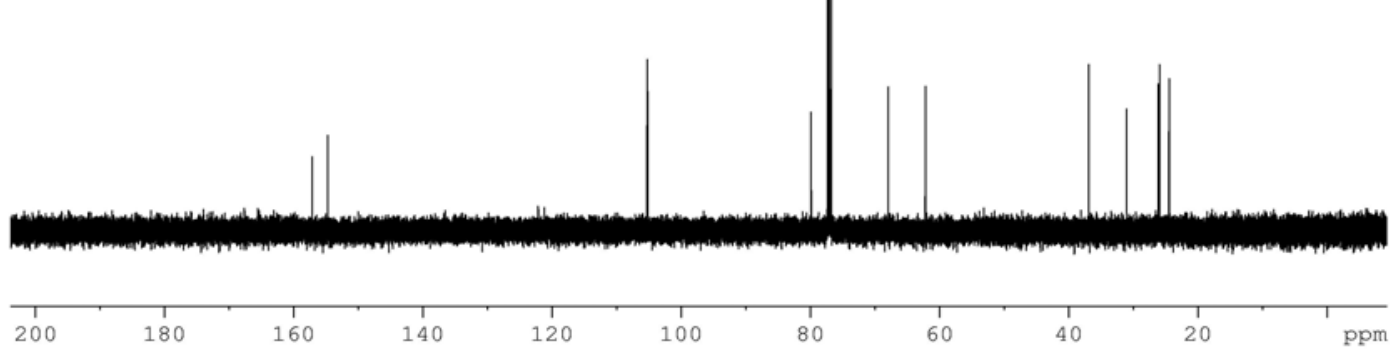




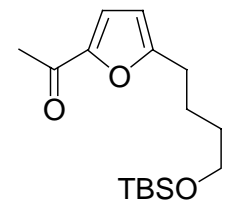

(500 MHz, $\mathrm{CDCl}_{3}$ )
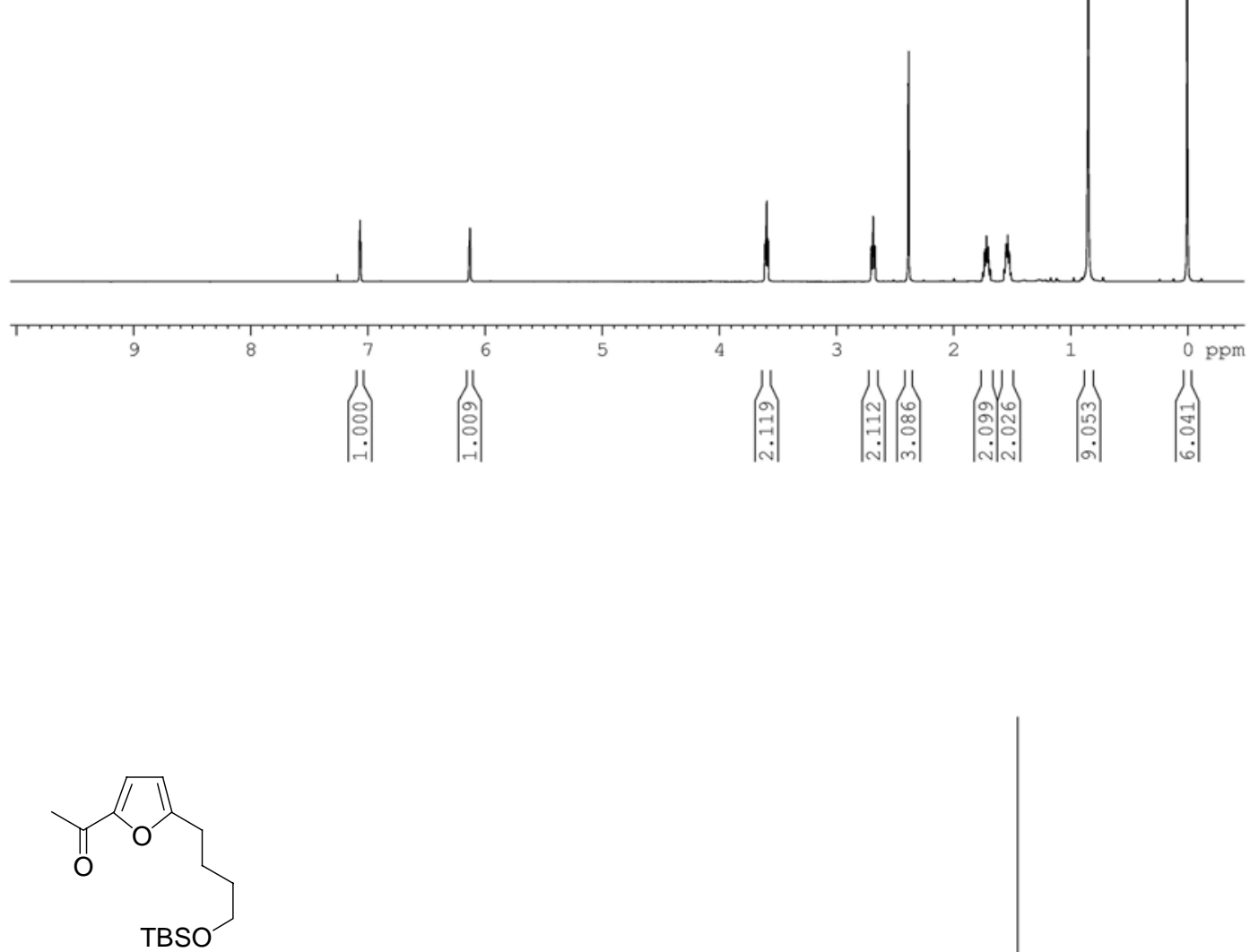

(125 MHz, $\mathrm{CDCl}_{3}$ )

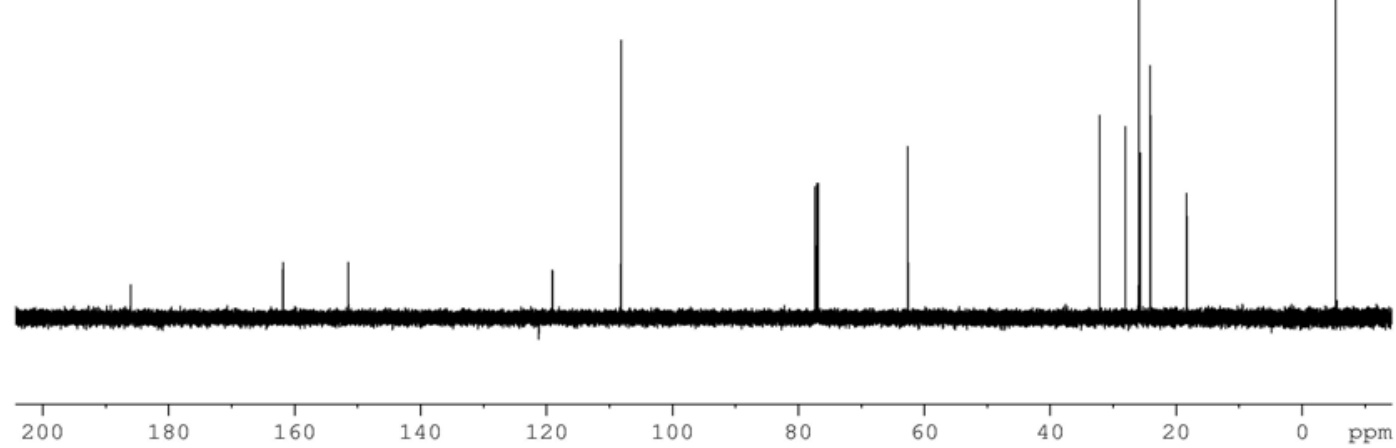



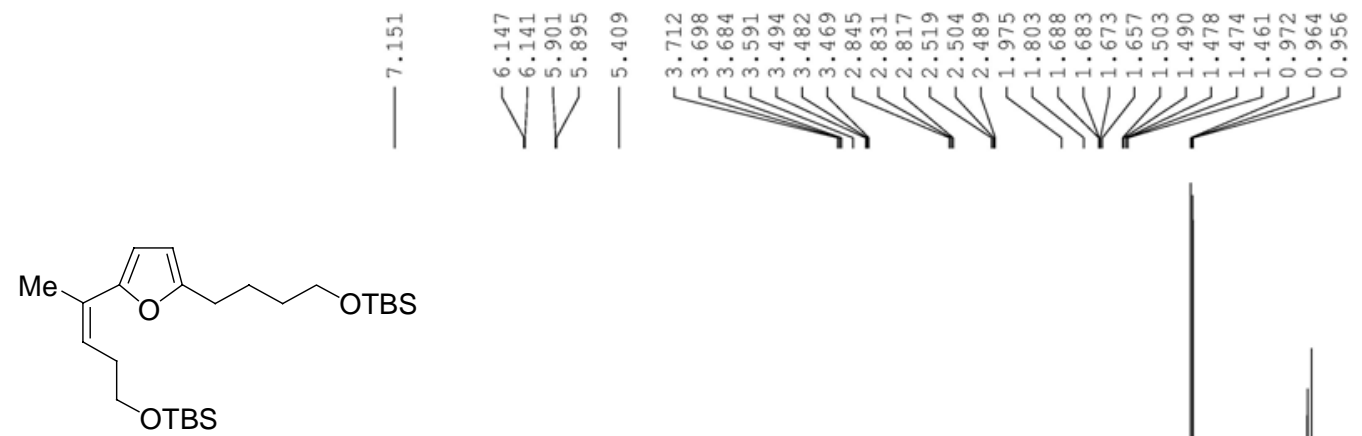

(500 MHz, $\mathrm{C}_{6} \mathrm{D}_{6}$ )
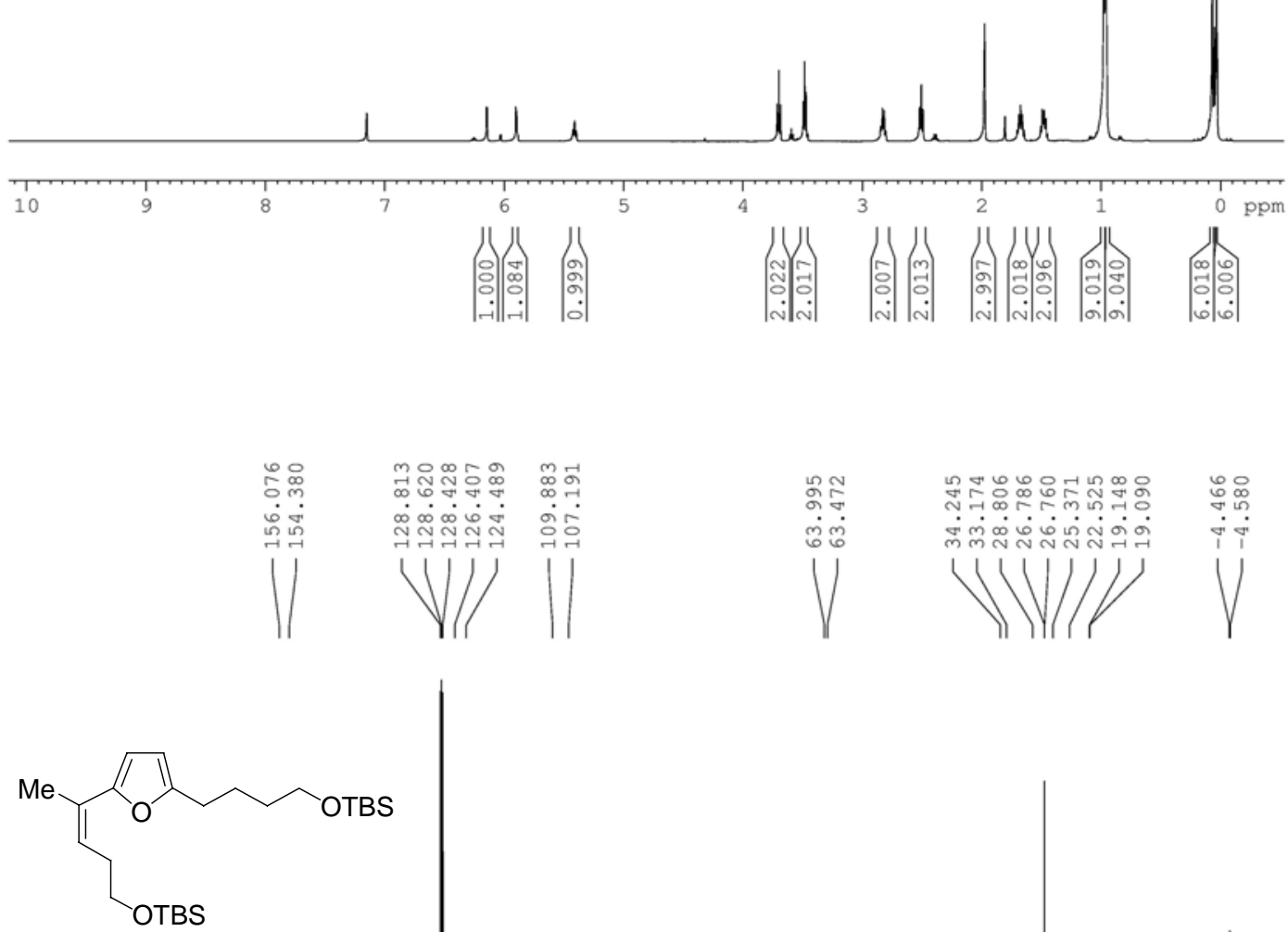

(125 MHz, $\mathrm{C}_{6} \mathrm{D}_{6}$ )
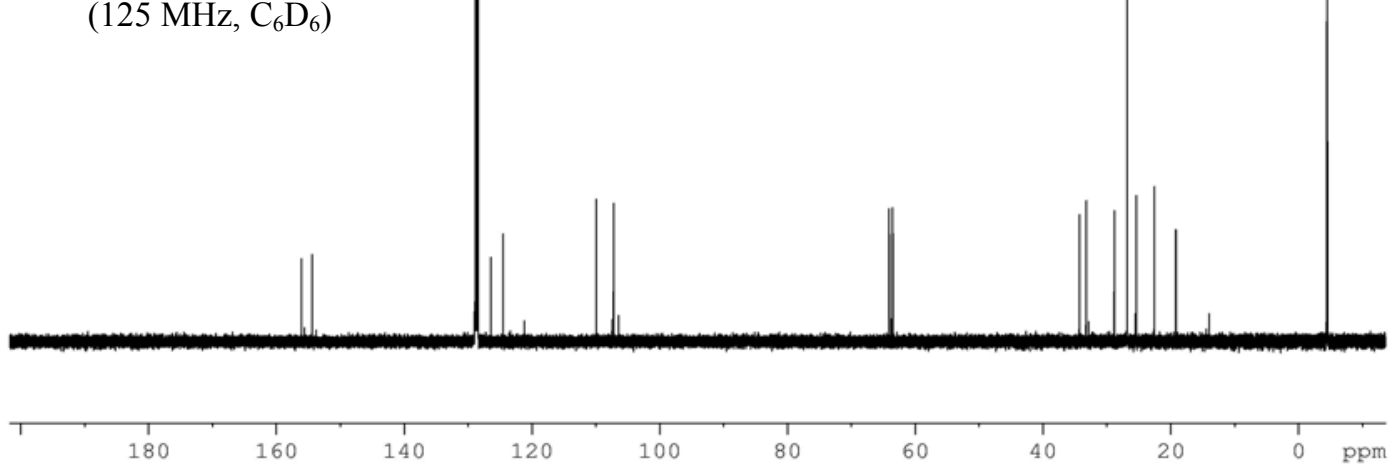


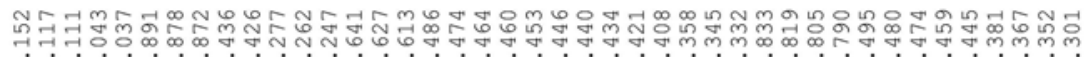

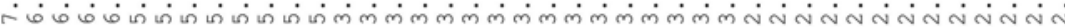

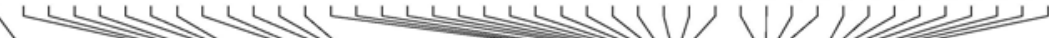
$\rightarrow 17$<smiles>C/C(=C/CCO)c1ccc(CCCCO)o1</smiles>

$\left(500 \mathrm{MHz}, \mathrm{C}_{6} \mathrm{D}_{6}\right)$
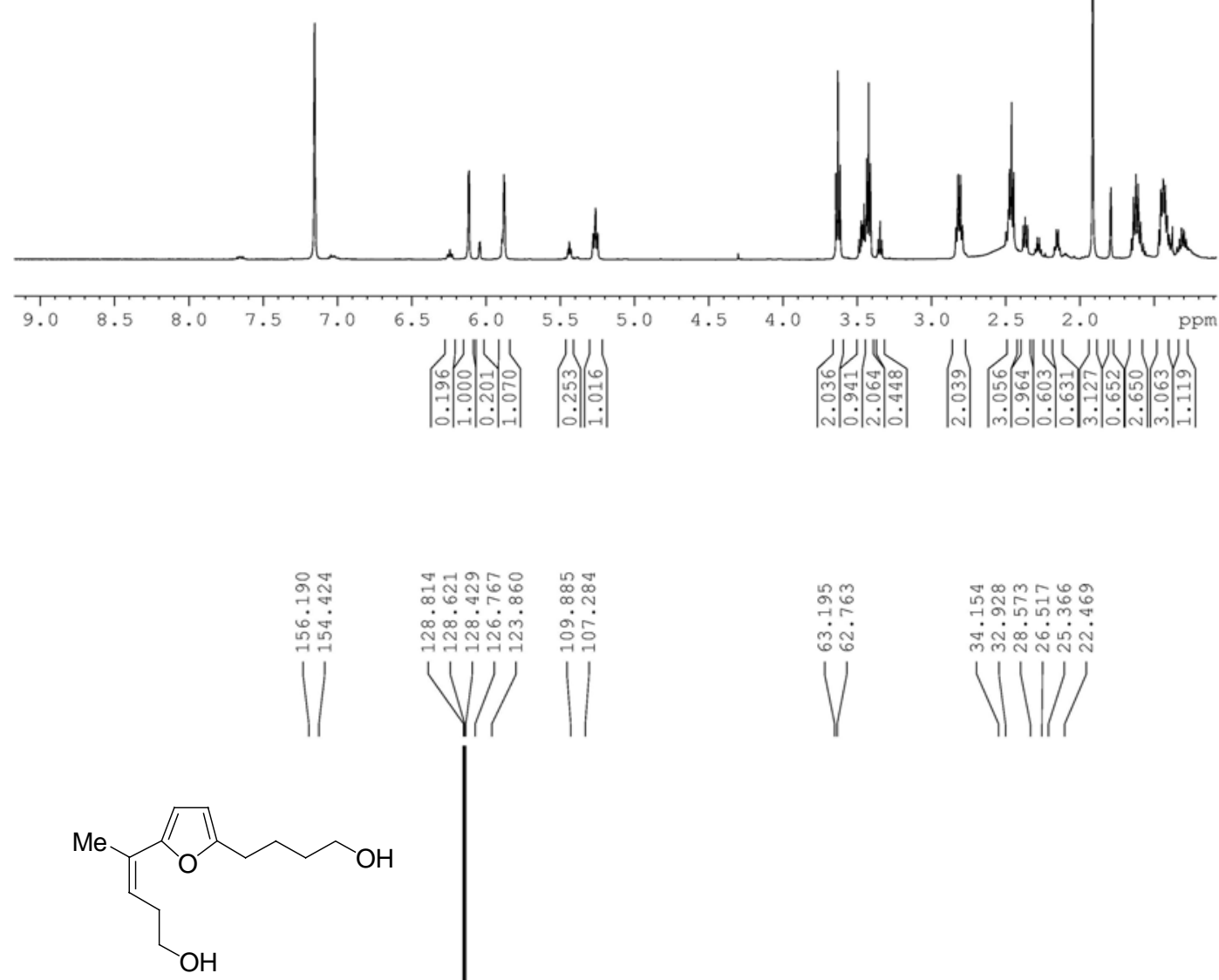

$\left(125 \mathrm{MHz}, \mathrm{C}_{6} \mathrm{D}_{6}\right)$
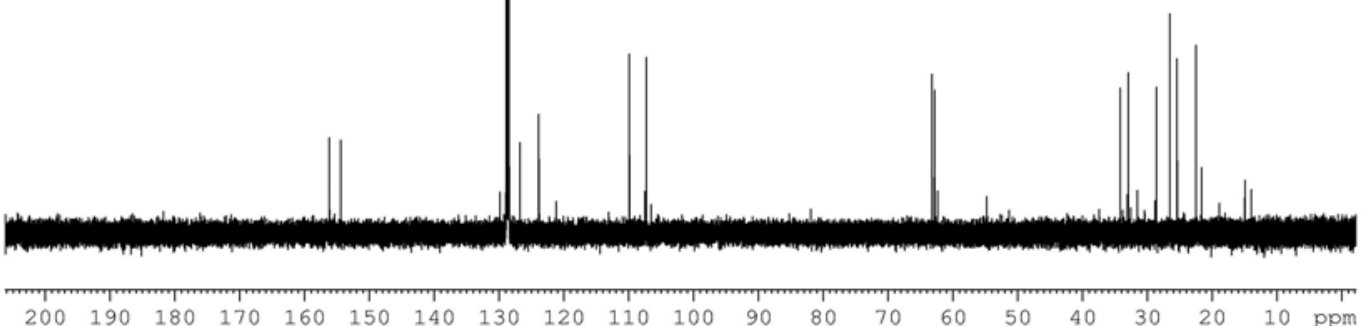


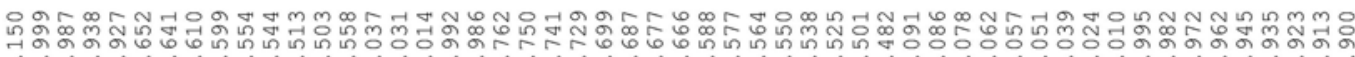
โก ம

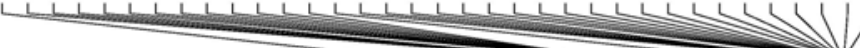

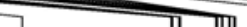

III

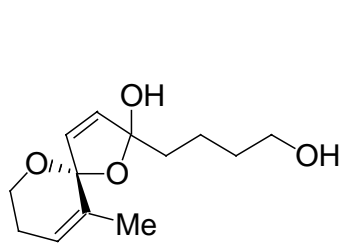

$\left(500 \mathrm{MHz}, \mathrm{C}_{6} \mathrm{D}_{6}\right)$
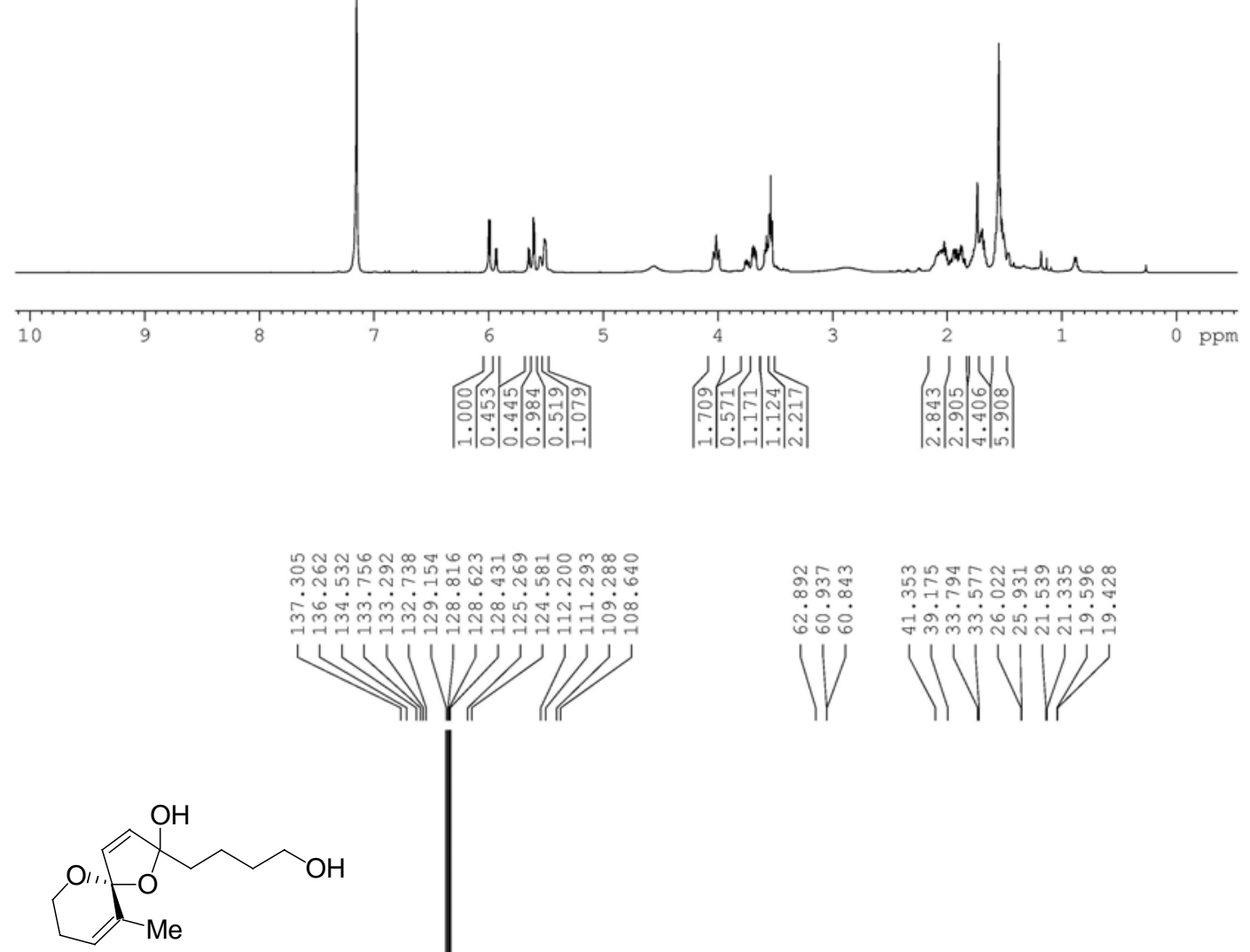

$\left(125 \mathrm{MHz}, \mathrm{C}_{6} \mathrm{D}_{6}\right)$
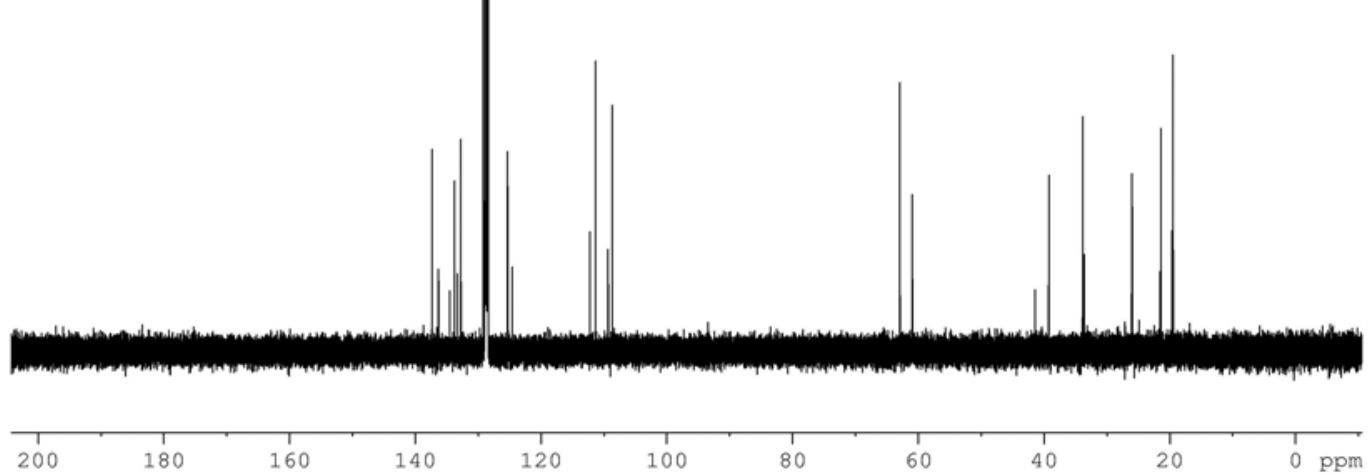


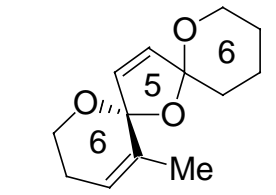

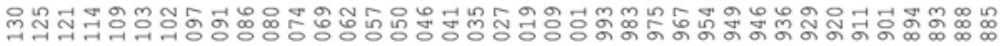

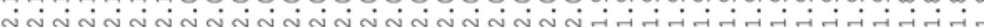
U
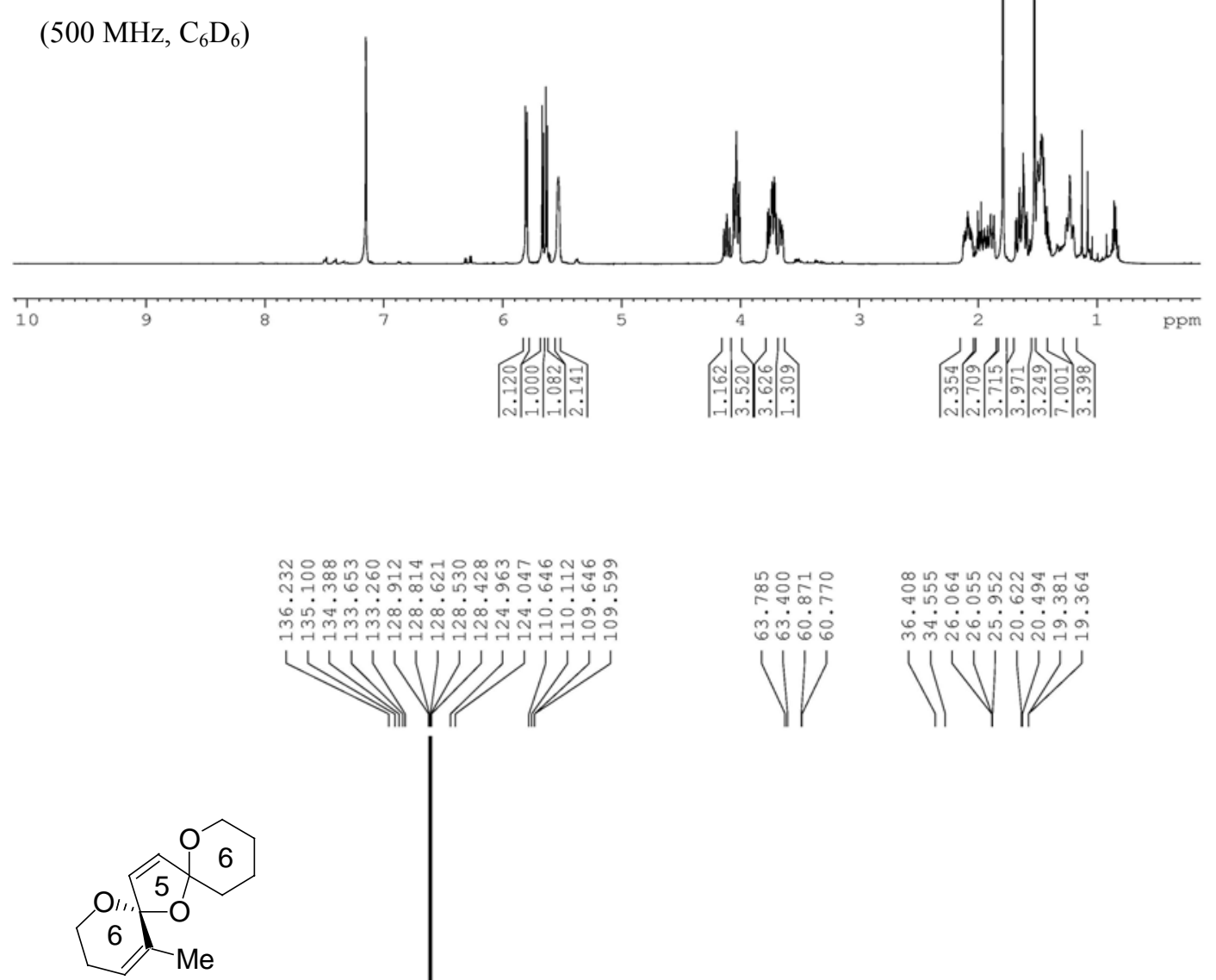

$\left(125 \mathrm{MHz}, \mathrm{C}_{6} \mathrm{D}_{6}\right)$

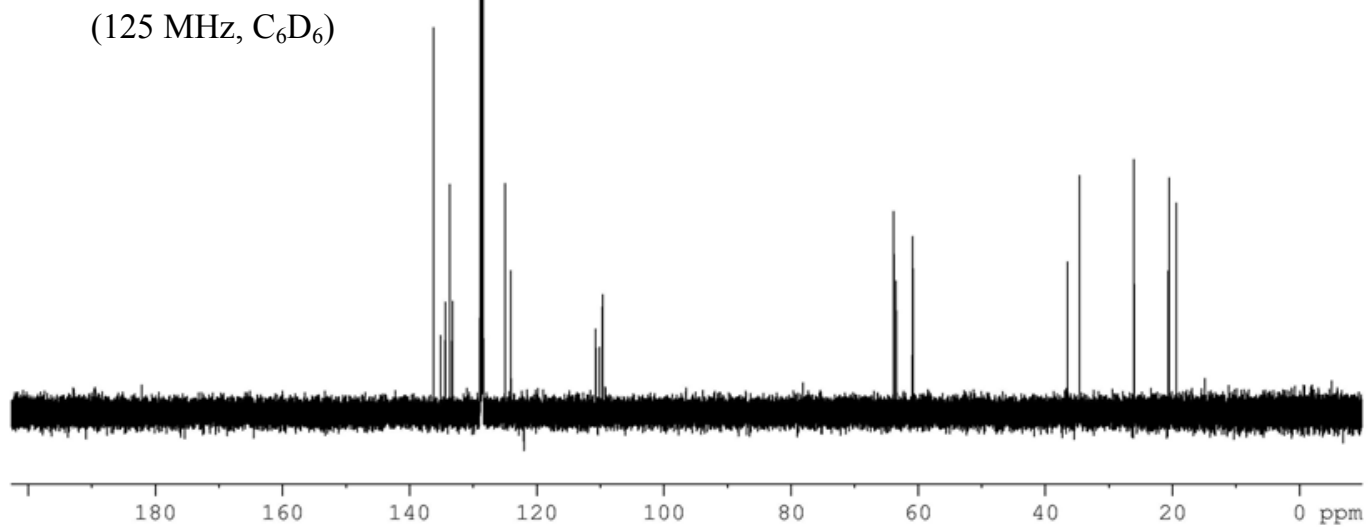

